\title{
Amplitudes of Pain-Related Evoked Potentials Are Useful to Detect Small Fiber Involvement in Painful Mixed Fiber Neuropathies in Addition to Quantitative Sensory Testing - An Electrophysiological Study
}

\author{
Niels Hansen ${ }^{1,2,3}$, Ann-Kathrin Kahn ${ }^{1}$, Daniel Zeller ${ }^{1}$, Zaza Katsarava ${ }^{4}$, Claudia Sommer ${ }^{1}$ \\ and Nurcan Üçeyler ${ }^{1 *}$
}

'Department of Neurology, University of Würzburg, Würzburg, Germany, ${ }^{2}$ Department of Neurophysiology, Ruhr-University Bochum, Bochum, Germany, ${ }^{3}$ Department of Epileptology, University of Bonn, Bonn, Germany, ${ }^{4}$ Department of Neurology, Evangelisches Krankenhaus, Unna, Germany

OPEN ACCESS

Edited by: Nick Spencer,

Flinders University, Australia

Reviewed by:

Brett A. Graham,

The University of Newcastle, Australia

Vaughan G. Macefield,

Western Sydney University, Australia

*Correspondence:

Nurcan Üçeyler

ueceyler_n@ukw.de

Specialty section:

This article was submitted to

Autonomic Neuroscience,

a section of the journal

Frontiers in Neurology

Received: 09 September 2015 Accepted: 09 November 2015

Published: 07 December 2015

Citation:

Hansen N, Kahn A-K, Zeller D,

Katsarava Z, Sommer $C$ and

Üçeyler N (2015) Amplitudes of

Pain-Related Evoked Potentials Are

Useful to Detect Small Fiber

Involvement in Painful Mixed Fiber

Neuropathies in Addition to

Quantitative Sensory Testing - An

Electrophysiological Study.

Front. Neurol. 6:244.

doi: 10.3389/fneur.2015.00244
To investigate the usefulness of pain-related evoked potentials (PREP) elicited by electrical stimulation for the identification of small fiber involvement in patients with mixed fiber neuropathy (MFN). Eleven MFN patients with clinical signs of large fiber impairment and neuropathic pain and ten healthy controls underwent clinical and electrophysiological evaluation. Small fiber function, electrical conductivity and morphology were examined by quantitative sensory testing (QST), PREP, and skin punch biopsy. MFN was diagnosed following clinical and electrophysiological examination (chronic inflammatory demyelinating neuropathy: $n=6$; vasculitic neuropathy: $n=3$; chronic axonal neuropathy: $n=2$ ). The majority of patients with MFN characterized their pain by descriptors that mainly represent C-fiber-mediated pain. In QST, patients displayed elevated cold, warm, mechanical, and vibration detection thresholds and cold pain thresholds indicative of MFN. PREP amplitudes in patients correlated with cold $(p<0.05)$ and warm detection thresholds $(p<0.05)$. Burning pain and the presence of par-/dysesthesias correlated negatively with PREP amplitudes $(p<0.05)$. PREP amplitudes correlating with cold and warm detection thresholds, burning pain, and par-/dysesthesias support employing PREP amplitudes as an additional tool in conjunction with QST for detecting small fiber impairment in patients with MFN.

Keywords: mixed fiber neuropathy, pain-related evoked potentials, A $\delta$ - and C-fibers, neuropathic pain, burning pain, quantitative sensory testing

\section{INTRODUCTION}

Painful polyneuropathies of different origin often affect large- and small-nerve fibers $(1,2)$ and are therefore termed mixed fiber neuropathies (MFN). Evaluating small fiber impairment can facilitate the differential diagnosis of painful MFN. Familial amyloid polyneuropathy is an example of MFN that may initially be asymptomatic (3) and where assessment of small fiber function may allow an 
early diagnosis. Small fiber tests could be appropriate in clinical course and analyzing therapy efficacy (4). The latter is of particular importance, since efficacious pharmacotherapy is disposable for several acquired neuropathies (5). Small thinly myelinated as well as unmyelinated nerve fibers (A $\delta$ - and C-fibers) can be assessed via neurological examination and quantitative sensory testing (QST) (6). However, both methods are subjective and very dependent on patients' cooperation. Since standard nerve conduction studies fail to measure the impairment of small-caliber nerve fibers, alternative objective means are needed. Nociceptive laser-evoked potentials (LEP) and contact heat-evoked potentials (CHEP) are suitable electrophysiological methods to detect functional impairment of small fibers in painful MFN (7). However, LEP and CHEP have drawbacks in clinical routine such as expensive equipment and complex procedures (8). The recording of electrically elicited pain-related evoked potentials (PREP) via concentric electrodes (9) is a useful, non-invasive, and handy method to detect small fiber impairment in early diabetes (10), human immunodeficiency virus (HIV) associated neuropathy (11), Fabry disease (12), and fibromyalgia syndrome (13). Neuropathic pain, pathological sural nerve conduction, and pathological PREP have been obtained in patients with hepatitis C- and HIV infection-associated sensory neuropathies $(11,14)$. PREP are thus suitable for detecting small fiber involvement in a variety of conditions characterized by small fiber pathology.

Painful MFN is a neuropathy encountered frequently in clinical routine, for example in patients with chronic inflammatory demyelinating neuropathy (CIDP). CIDP patients often have small fiber impairment in addition to large fiber affection (15). Neuropathic pain in large fiber neuropathy patients indicates but does not ascertain the involvement of small nerve fibers $(16,17)$. To accelerate diagnosis and treatment, and to monitor the time course of small nerve fiber degeneration or pharmacotherapeutic efficacy in MFN patients, a simple and objective tool to determine small fiber involvement is required.

We therefore investigated the usefulness of PREP in assessing small fiber involvement in painful MFN. We additionally utilized QST as a standard instrument to retrieve small fiber function in neuropathy patients and compared PREP and QST data in MFN. We hypothesized that PREP amplitudes would be reduced and PREP latencies delayed particularly when elicited from the feet in patients with additional small fiber affection.

\section{BACKGROUND}

\section{Patients and Subjects}

In this study, eleven patients with painful MFN (median age $=61$ years, range: $37-83$ years, three females and eight males) were prospectively enrolled in parallel to the recruitment of healthy control subjects. The diagnosis was made when clinical signs of large fiber affection as well as neuropathic pain coincided. The recruitment of patients was performed between 2009 and 2012 at the Department of Neurology, University of Würzburg. Inclusion criteria consist of female and male patients at the age of $\geq 18$ years, confirmed diagnosis of painful motor or sensory polyneuropathy (by medical report, neurological examination, standard neurophysiology). Exclusion criteria were: pain of other than neuropathic origin (e.g., dermatologic, orthopedic) or abnormal blood investigations not related to the neuropathy. A robust and reproducible PREP response was a prerequisite for including MFN patients in our study, as we were dedicated to delineating the differences between PREP latencies and amplitudes in patients and controls.

We compared our data with those from ten healthy age- and gender-matched controls recruited during our study (median age $=55$ years, range: $26-67$ years; four females and six males). Inclusion criteria for control subjects comprised the following items: $\geq 18$ years, unremarkable sural nerve conduction, no neuropathy and no report of pain (neuropathic pain or other causes of pain). The age and gender did not vary significantly between patients and control subjects ( $p>0.05$ each). The performance of the study was in agreement with the Declaration of Helsinki. The study was authorized by the Würzburg Medical Faculty Ethics Committee. Recorded informed consent was received from all participants of the study.

\section{Neurological Investigation, Pain and Depression Questionnaires, Neuropathy Scales}

Every patient was subject to a neurological examination and was investigated by employing the German translations of pain and depression questionnaires as well as neuropathy scales. Severity of neuropathy was estimated using a variation of the Neuropathy Deficit Score (NDS) and the Neuropathy Symptoms Score (NSS) $(18,19)$. The Neuropathic Pain Symptom Inventory (NPSI; $24-\mathrm{h}$ recall) $(20,21)$ assesses neuropathic pain characteristics and intensity; the resulting sum score goes from 0 (no pain) to 1 (maximum pain). The McGill pain questionnaire (22) was employed to characterize patients' pain by distinct affective and sensory descriptors. Patients' answers to each of the 78 questions ranged between 0 (inappropriate) and 4 (fully appropriate). To discriminate between $\mathrm{A} \delta$ - and $\mathrm{C}$-fiber excitation attributed pain, a rating test utilizing the adjectives "dull," "pressing" and "pricking" from the McGill pain questionnaire was applied according to Beissner (23). Pain was attributed to C-fiber excitation if the sum of selecting "dull" or "pressing" was higher than that of choosing "pricking" (23). Pain magnitude (score based on three pain intensity items) and impact of pain (score of three items on pain perturbation with everyday life activities) was investigated by the Graded Chronic Pain Scale (GCPS; an altered version consisting of a 4-week recall) (24).

To additionally identify potential depressive symptoms, we utilized the Center for Epidemiologic Studies Depression Scale ("Allgemeine Depressionsskala," ADS; 1-week recall) (25). The maximum ADS score is amounted to 60 ; a total score $\geq 16$ is expected to be relevant in clinical practice.

To diagnose CIDP the Inflammatory Neuropathy Cause and Treatment (INCAT) criteria (26) were applied; the EFNS/PNS criteria were used to diagnose non-systemic vasculitic neuropathy (27).

\section{Quantitative Sensory Testing}

All patients and healthy controls underwent QST. Following current recommendations (28-31), we log-transformed the QST 
parameters and calculated $z$-scores, which allows normalization of the patient and control data to the data of the controls (mean and SD), i.e., also the data of the ten controls were referenced to themselves.

\section{Parameters}

We performed QST in each study participant on the dorsum of the foot following the standardized protocol of the German Research Network Neuropathic Pain (Deutscher Forschungsverbund Neuropathischer Schmerz, DFNS) and using a calibrated device (Somedic, Hörby, Sweden) (28, 29). We assessed the successive determinants: cold detection threshold (CDT), cold pain threshold (CPT), warm detection threshold (WDT), heat pain threshold (HPT), capability to identify temperature alterations (thermal sensory limen, TSL), mechanical detection threshold (MDT), mechanical pain sensitivity (MPS), mechanical pain threshold (MPT), pressure pain threshold (PPT), and vibration detection threshold (VDT).

In brief: for CDT and HDT, a thermode was positioned above the dermis and the temperature lowered or raised starting at $32^{\circ} \mathrm{C}$ $\left(\min .10^{\circ} \mathrm{C}, \max .50^{\circ} \mathrm{C}\right.$ ). Each control subject or patient pushed a knob when the temperature he or she sensed was cold or warm, respectively. CPT and HPT were assessed accordingly. TSL was determined by alternately changing the thermode's temperature beginning at $32^{\circ} \mathrm{C}$; the subject pushed a button to indicate when a change in temperature was felt. MDT was determined by stimulating the skin with calibrated von Frey filaments; the examination started with a von Frey filament of $16 \mathrm{mN}$ and the intensity reduced or increased in the filament being sensed by the subject defining the MDT (range 0.25-512 mN). Calibrated pinpricks (range $0.25-512 \mathrm{mN}$ ) were applied to determine the MPT; to determine the MPS, the subject was stimulated with a pin-prick and thereafter with a brush, a Q-tip or a cotton ball; the subject then rated the degree of strength of the pinprick stimulus on a chart from 0 to 100 . The VDT was determined using a tuning fork. A calibrated algesiometer (Wagner Instruments, USA) was used to assess the PPT.

\section{Assessment}

For group analysis, patients' data were compared with data from 10 healthy control subjects following the same protocol at our department. According to current recommendations (28-31), QST raw values were log-transformed to reach normal distribution (CDT, MDT, MPS, PPT, TSL, WDT). The CPT, HPT, and VDT parameters were not log-transformed $(28,29)$, as the ratio for raw data to log-transformed data did not cross a factor of three, and as one prerequisite for log-transformation (i.e., determination of the exact zero-threshold of the respective parameter) was unfulfilled (29). With these data, we calculated a $z$-score utilizing the formula: $z$-score [(value of the patient - mean value of control subjects)/SD of control subjects]. Calculating the $z$-score allows the normalization of patient data to the control group. Positive $z$-scores reveal a gain of function whereas negative $z$-scores point to a loss of function in comparison to the controls. Since in this formula the controls' QST values are normalized to their own mean and SD, the controls' QST $z$-score values result in zero with a SD of one. Since the MPS examination may result in "zero," we applied the Bartlett procedure $(31,32)$ and added a constant of 0.1 to the MPS results for statistical reasons (33). $Z$-values below or above \pm 1.96 were determined as aberrant $(30,34)$.

For individual assessments of QST results, we used published normative data (30). The controls' QST values were within the extent of released QST data from healthy control groups $(12,13,35,36)$.

\section{Electrophysiological Assessment}

All patients and healthy controls underwent nerve conduction studies. The right sural and tibial nerves in patients and the right sural nerves in healthy controls were examined utilizing electrodes according to standard procedures (37) to determine large fiber polyneuropathy. Findings were compared with our laboratory normal values for adults. Our laboratory normal values consist of an antidromic sural nerve action potential (SNAP) amplitude $\geq 10 \mu \mathrm{V}$ for age $<65$ years and $\geq 5 \mu \mathrm{V}$ for age $>65$ years. The normal values for the sural nerve conduction velocity ( $\mathrm{NCV}$ ) were $>40 \mathrm{~m} / \mathrm{s}$ for all age groups. The tibial nerve compound motor action potential (CMAP) was composed of values $\geq 10 \mathrm{mV}$ and the tibial $\mathrm{NCV} \geq 40 \mathrm{~m} / \mathrm{s}$ for all age groups.

\section{Pain-Related Evoked Potentials}

All patients and healthy controls underwent PREP derived from $\mathrm{Cz}$ via a needle electrode sited subcutaneously and referenced to connected ear lobes (A1-A2, international 10-20 system) as formerly mentioned in detail (13). Signal Software (Version 2-16, Cambridge Electronic Design, Lt., Cambridge, UK) was used. The potentials were elicited via electrical stimulation with a constant current stimulator (DS7A, Welwyn Garden City, UK) at the right and left foot (dorsum) employing planar concentric electrodes attached to the skin (Inomed Medizintechnik $\mathrm{GmbH}$, Lübeck, Germany). This stimulation paradigm consists of 20 triple pulses at twofold intensity of each pain threshold lasting $0.5 \mathrm{~ms}$, a triple pulse interval of $5 \mathrm{~ms}$ and random inter-stimulus interval of 15-17 s. The recording set-up comprises the following adjustments: gain: $\times 5000$, bandwidth: $1 \mathrm{~Hz}-1 \mathrm{kHz}$, digitalization sampling rate: $2.5 \mathrm{kHz}$ and sweep length: $400 \mathrm{~ms}$. Single pain threshold was ascertained by stimulating twice with current intensities that rose and fell until the person remarked a pin-prick perception. The MATLAB software (Version 7.7.0471, Ismaning, Germany) was utilized to survey averaged curves ( $n=20$ single sweeps) for reproducible N1- (i.e., first negative peak), P1- (i.e., subsequent positive peak) latencies, and peak-to-peak amplitudes (PPA). Every curve was analyzed off-line via an examiner blinded to the diagnosis on coded data files. Recordings containing technical or biological artifacts were excluded from the analysis. Only those recordings were included that showed robust and reproducible potentials. Additional exclusion criteria for PREP recordings were: history of epilepsy, cardiac pacemakers, and deep brain stimulators.

\section{Skin Biopsies}

All patients underwent skin punch biopsies with a size of $5 \mathrm{~mm}$ (punch device: Stiefel, Offenbach, Germany) obtained to assess intra-epidermal nerve fiber density (IENFD). The locus for the 
removal of the biopsies was the lower extremity $10 \mathrm{~cm}$ proximal to the lateral malleolus. The skin probes were treated as specified formerly (38). Immunoreactions of the individual sections were caused by antibodies to protein-gene product (PGP) 9.5 (Ultraclone limited, Isle of Wight, UK, primary antibody; 1:800). The PGP 9.5 antibodies were combined with goat anti-rabbit IgG marked with cyanine 3.18 fluorescent sample (Amersham, USA, Cy3, secondary antibody; 1:100) (12). The IENFD was determined by an investigator blinded to the individual sample's identification according to released approaches (39).

\section{Statistical Analysis}

We used Statistica 6410 (Copyright 1984-2011, Tulsa, OK, USA) for statistical analysis and Sigma Plot 11.0 for Windows (Copyright 2008, San Jose, California, USA) for graph creation. Normally distributed data was subjected to the Shapiro-Wilk test. To compare data without normal distribution, we applied the non-parametric Mann-Whitney $U$ test, and the parametric student's $t$-test to compare data with normal distribution. Data without normal distribution are shown as median and range (demographic data, questionnaires, sural nerve electrophysiology data), whereas data that show a normal distribution are illustrated as mean \pm SD (PREP as well as QST data). For comparison with previously published data, the McGill pain questionnaire results are depicted as mean \pm SD. For correlation analysis, the Pearson Product Moment correlation was utilized. The level of significance was set to $p<0.05$.

\section{RESULTS}

\section{Questionnaire Results, Clinical Findings, and Standard Nerve Conduction Results}

Our study cohort's demographics, neuropathy scale scores, questionnaire results, and electrophysiology data are summarized in Table 1. Diagnoses and characteristics documented during the neurological examination are listed in Table S1 in Supplementary Material. Eleven patients with neuropathic pain (i.e., pain of $\geq 4 / 10$ on a numeric rating scale, NRS) and signs of large-fiber involvement were classified as patients with painful MFN. The controls comprised ten healthy subjects from our department not presenting any neuropathy, neuropathic pain, or other causes of pain. The patient group consisted of six patients with CIDP (26), three patients with vasculitic neuropathy (27), and two patients with chronic axonal neuropathies. Neurological examination of the lower limb revealed pathological findings in all patients, whereas the healthy controls revealed no clinical abnormalities: 11/11 (100\%) patients reported hypoesthesia to touch, 6/11 (54\%) patients had thermal hypoesthesia, 11/11 (100\%) patients had pallhypoesthesia, 7/11 (64\%) patients presented pareses, and 10/11 (91\%) patients had hyporeflexia. Autonomic dysfunction was present in 3/11 (27\%) patients. One to two patients each $(9-18 \%)$ reported disturbance in micturition or nycturia, cardiac arrhythmia, diarrhea, erectile dysfunction, and esophageal dysmotility. Nerve conduction studies of the tibial nerve revealed reduced CMAP in 10/11 (91\%) patients and slowed NCV in 9/11 (82\%) patients. Either the CMAP or
TABLE 1 | Demographic, questionnaire, and neurophysiological data of patients with mixed fiber neuropathy and controls.

MFN Controls $p$-value
patients

\section{Demographic data}

Number (male, female)

Median age years (range)

Median disease duration in years

(range)

\section{Questionnaires}

Current pain intensity (NRS; 0-10)

Median NPSI sum score

Median GCPS sum score (intensity)

Median GCPS sum score

(impairment)

Median ADS score

Median NDS sum score (0-272)

Median NSS sum score (0-18)

Electrophysiology

Sural nerve

Sensory nerve action potential $(\mu \mathrm{V})$

Nerve conduction velocity $(\mathrm{m} / \mathrm{s})$

$\begin{array}{ccc}11(8,3) & 10(6,4) & \text { ns } \\ 61(37-83) & 55(26-67) & \text { ns } \\ 2(0.1-4.5) & & \end{array}$

ns

n
ADS, Allgemeine Depressionsskala; GCPS, Graded Chronic Pain Scale; MFN, mixed fiber neuropathy; NDS, Neuropathy Disability Scale; NPSI, Neuropathic Pain Symptom Inventory; ns, not significant; NSS, Neuropathy Symptom Scale; NRS, Numerical Rating Scale.

NCV was abnormal in all patients. Electrophysiological assessment of the sural nerve (Table 1) showed reduced SNAP in $4 / 6(67 \%)$ and no SNAP in $1 / 6(17 \%)$ patients aged $<65$ years and reduced SNAP in $4 / 5(80 \%)$ and no SNAP in $1 / 5(20 \%)$ of patients aged $>65$ years. NCV of the sural nerve was abnormal in $4 / 11(36 \%)$ patients. In contrast, the control subjects' sural nerve conduction examinations were normal. 7/11 (64\%) of patients received continious analgesic treatment (pregabalin $n=5$, gabapentin $n=2$ ), whereas the controls received no analgesic therapy.

\section{QST Revealed Large and Small Fiber Impairment}

The detection thresholds of MFN patients as well as control subjects are illustrated in Table S2 in Supplementary Material. MFN patients had more elevated CDT and WDT than the control subjects ( $p<0.05$, Figure 1) translating to reduced cold and warm detection capacities and indicating functionally impaired A $\delta$ - and C-fibers. MFN patients thus displayed warm and cold hypoesthesia compared to the controls. Furthermore, patients' MDT and VDT were higher than those of controls $(p<0.05$, Figure 1). These alterations suggest impaired Aß-fiber function and can be termed mechanical hypoesthesia compared to the controls. The increased HPT in MFN patients $(p<0.05$ vs. controls, Figure 1) is compatible with impaired small fiber dysfunction and is called heat hypoalgesia. In healthy control subjects, the QST values fell within the confidence interval $( \pm 1.96)$, indicating normal values. 


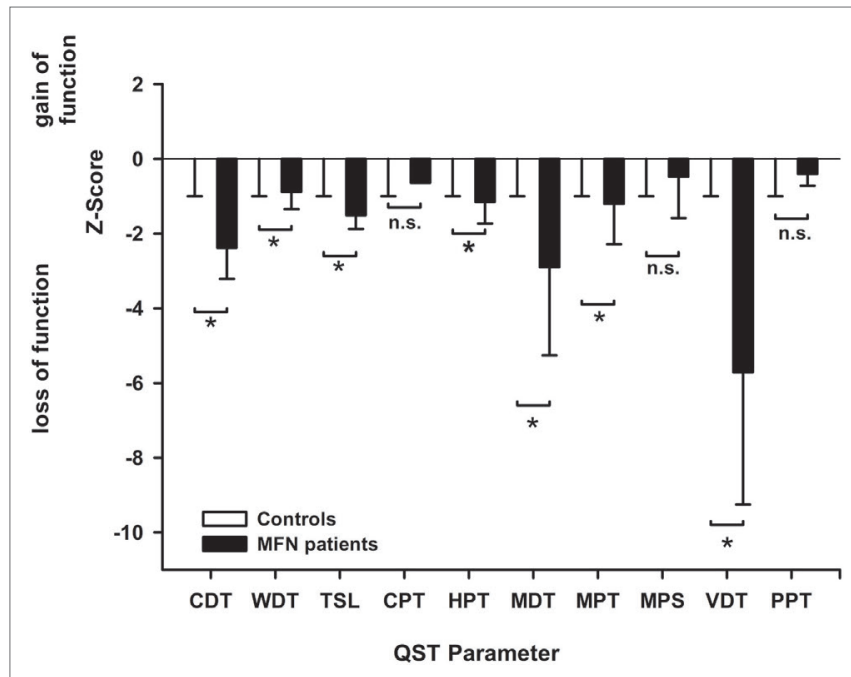

FIGURE 1 | Sensory profile of patients with mixed fiber neuropathy (MFN) and controls obtained by quantitative sensory testing (QST). The bar graphs depict the $z$-scores at the dorsal foot in MFN patients contrasted to healthy control subjects. Healthy controls are shown by white bar graphs, whereas MFN patients are represented by black bar graphs. The $z$-scores of healthy controls are zero with a SD of one due to the calculation of $z$-score (see methods). This is the reason why the white bar graphs are not seen and disappear in the zero-line. $z$-scores $<0$ indicate a loss of function, $z$-scores $>0$ exhibits a gain of function. MFN displays impaired cold (CDT), warm (WDT), mechanical (MDT), and vibration detection thresholds (VDT) as well as cold pain thresholds (CPT) compared to controls $(p<0.05)$ indicating deafferentation of small and large fibers. NB: Patients' CPT was $10^{\circ} \mathrm{C}$ which is the cut-off value for CPT assessment during QST. Therefore, the CPT value does not have an error bar. Abbreviations: CPT, cold pain threshold; HPT, heat pain threshold; ability to detect temperature changes (thermal sensory limen, TSL); MPT, mechanical pain threshold; MPS, mechanical pain sensitivity; PPT, pressure pain threshold (PPT); VDT, vibration detection threshold. Data are expressed as mean and SD. ${ }^{*} p<0.05$

\section{Altered Thermal Perception in Patients with MFN Correlates with PREP Amplitudes}

Stimulus intensities used to elicit PREP from the feet were not different between MFN patients and control subjects (Table 2). We analyzed pooled data (N1, P1, and PPA as well as stimulus intensities) from both sides of the lower legs since no side difference had been observed. N1 and P1 latencies as well as PPA amplitudes of PREP were not different between MFN and control subjects, except for a tendency toward prolonged N1/P1 latencies and reduced PREP amplitudes in MFN (Table 2; Figure 2 shows a representative example of a PREP trace of a control subject). Analysis of PREP parameters in the largest subgroup of CIDP patients $(n=6)$ compared to controls revealed no intergroup difference. Between PREP PPA induced at the feet and thermal perception thresholds (CDT, WDT, TSL) of MFN patients a positive correlation was encountered (Figures 3A-C). We noted a positive correlation for CDT in MFN patients $(r=0.71, p<0.05$, Figure 3A), as well as for WDT ( $r=0.63, p<0.05$, Figure 3B) and TSL $(r=0.67, p<0.05$, Figure $3 \mathrm{C})$. We found no correlation for CDT, WDT, and TSL with PREP PPA in control subjects
TABLE 2 | Pain-related evoked potentials elicited at the foot and stimulus intensity data of patients and controls.

\begin{tabular}{lccc}
\hline Parameter & MFN patients & Controls & p-value \\
\hline N1 latency (ms) & $176 \pm 47$ & $162 \pm 31$ & 0.46 \\
P1 latency (ms) & $228 \pm 44$ & $210 \pm 47$ & 0.39 \\
PPA $(\mu \mathrm{V})$ & $30 \pm 15$ & $39 \pm 31$ & 0.45 \\
Current intensity $(\mathrm{mA})$ & $2.07 \pm 0.37$ & $1.8 \pm 0.4$ & 0.15 \\
\hline
\end{tabular}

ns, not significant; PPA, peak-to-peak amplitudes.

(Figures 3D-F). CPT, and HPT $z$-scores as functions of small fibers and MDT, MPT, VDT, and PPT $z$-scores reflecting large fiber function did not correlate with PREP PPA in patients with MFN or in the controls. Moreover, we detected no correlations between PREP, sural nerve NCV, and sural nerve SNAP amplitude and controls. Burning pain quality and the presence of par- or dysesthesias assessed by the respective NPSI subscores correlated negatively with PREP PPA in MFN patients $(r=-0.68, p<0.05$, Figures $4 \mathbf{A}, \mathbf{B})$. We observed no correlation between paroxysmal and evoked pain and PREP PPA in patients with MFN. Moreover, IENFD showed no correlation with the intensity of burning pain and the existence of par- or dysesthesias in the NPSI subscores of patients with MFN (Figures 4C,D). Pain intensity elicited by the electrical stimulus did not correlate with the PREP PPA in MFN patients and controls. Furthermore, the IENFD failed to correlate with either PREP latencies or amplitudes. In particular, we observed no correlation between the loss of IENFD in distal skin biopsies and burning pain or with par- and dysesthesias in MFN patients. In addition, we found no correlation between electrical stimulus intensity and IENFD.

Analyzing the NPSI subscores, burning pain was the most frequently reported pain quality in patients with MFN (64\%), whereas less than half of the patients experienced pressure, evoked, or paroxysmal pain (36-47\%; see Table S3A in Supplementary Material). In contrast, our healthy control subjects reported no burning, pressure, evoked or paroxysmal pain. In the McGill pain questionnaire, MFN patients characterized their pain using sensory and affective pain descriptors, whereas the controls reported no pain (see Table S3B in Supplementary Material). To define the predominance of $\mathrm{A} \delta$ - or $\mathrm{C}$-fiber-mediated pain, the total amount of the "dull" or "pressing" pain descriptor selections was calculated from the McGill pain questionnaire data (23). This sum was higher than that of the selection "pricking" pain in 8/9 MFN patients with CIDP and vasculitic neuropathy who had completed the questionnaire correctly, indicative of C-fiber-mediated pain (2/11 patients refused to fill in the McGill questionnaire).

\section{DISCUSSION}

Our results demonstrate that, in addition to QST, PREP amplitudes may provide a useful parameter reflecting functional small fiber impairment in patients with painful MFN. This observation is underpinned by the strong correlation among increased thermal perception thresholds and reduced PREP amplitudes in patients with MFN, and by the correlation between ongoing burning pain as well as par- and dysesthesias and reduced PREP amplitudes. 


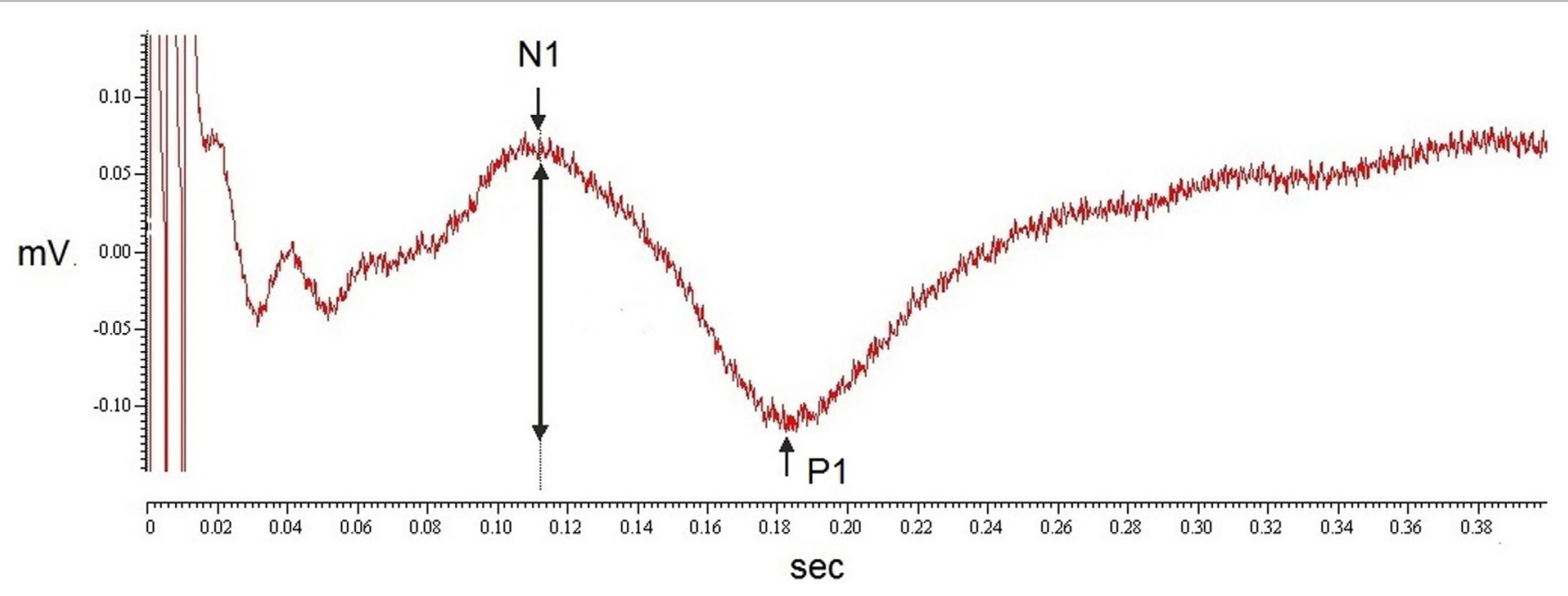

FIGURE 2 | A representative trace of a PREP recording in a control subject. The figure shows an example of a representative PREP curve from a healthy control subject. The N1 and P1 latency are indicated by arrows; the peak-to-peak amplitude is indicated by a two-sided arrow.

Similar results have been obtained using other methods in small fiber neurophysiology. Nociceptive N2/P2 latencies were delayed and CHEP and LEP amplitudes reduced or even absent in MFN compared to healthy controls (7). The N2/P2 amplitude of LEP was reduced in patients with Charcot-Marie-Tooth disease type 1A (40). Two studies showed prolonged N1/P1 latencies and reduced PREP amplitudes in 13 patients with hepatitis $C$ versus 28 healthy controls and nine patients with HIV-associated MFN compared to nine controls $(11,14)$. In our study, we detected neither prolonged N1/P1 latencies nor reduced PREP amplitudes in MFN patients compared to controls. This may be due to methodological differences (CHEP, LEP vs. PREP) and cohort size, and to our patient group's heterogeneity.

Our MFN cohort consisted of different diagnostic subgroups (CIDP, vasculitic neuropathy, chronic axonal neuropathy). It is known that the type of neuropathy can affect cerebral painassociated potentials; e.g., LEP may be absent in metabolic or toxic neuropathies (41). Thus, future large-scale studies enrolling patients from different MFN subgroups are necessary to determine whether the trend toward prolonged PREP latencies and reduced amplitudes is relevant in some types of MFN and masked by other subgroups of MFN patients.

Moreover, due to the small sample size of cohorts, demographic factors such as gender and age cannot be investigated by this study. It is of great interest to determine the influence of these factors on PREP parameters in a future large-scale study.

Due to the short anode-to-cathode distance design of the concentric electrode nociceptive afferents in the superficial skin are activated. By this electrode arrangement, a high current intensity is gained when choosing low current stimulation. Furthermore, the electrical stimulation caused a pin-prick sensation that is a phenomenon associated with the excitation of $A \delta$-fiber nociceptors (42-44), and the NCVs achieved by stimulating these concentric electrodes fall within the range of A $\delta$-fibers $(10,12,45,46)$. No PREP were detected at up to $2.5 \mathrm{~mA}$ of electrical stimulation intensity after topical application of lidocaine, which leads to a selective loss of thermal and pain sensation, but does not affect tactile sensation (47). These findings suggest that PREP are mediated by small-caliber nerve fibers (47). The response of the blink reflex was inhibited by $90 \%$ after topical application of lidocaine, indicating that the blink reflex response is contaminated by the co-activation of Aß-fibers by $10 \%$ (9). Co-activation of Aß-fibers can be induced by the concentric electrode $(48,49)$ indicated by latency gaps between PREP and LEP that are larger than the thermoreceptor activation time of $40 \mathrm{~ms}(48,50)$. However, in this study, the amplitudes, morphology, and topographic localization were similar between PREP and LEP (48) suggesting that PREP amplitudes might be the more specific nociceptive marker than PREP latencies. The coactivation of Aß-fibers might be caused by employing a current intensity between 3 and $5 \mathrm{~mA}$ applied via the concentric electrode (49). Therefore, particular care must be taken to avoid additional Aß-fiber activation (e.g., by keeping stimulus intensities below $2.5 \mathrm{~mA}$ ) during PREP measurements that are unnecessary when applying LEP (48). In our study, the electrical stimulation intensity was tuned below $2.5 \mathrm{~mA}$, so that large and medium-sized fibers might have been unspecifically stimulated to a small but irrelevant extent.

Therefore, this factor probably does not account for the lack of a difference between our patients' and controls' PREP parameters. The characterization of late components of PREP needs future studies. This may help to differentiate between the excitation of different fiber types similar to the reported early and late components of LEP indicating A $\delta$ - or C-fiber excitation (51).

Another issue is the potential influence of analgesic drugs on PREP parameters. As experimental in vitro evidence suggests, nociceptive transmission in the dorsal horn is altered by anticonvulsant drugs such as gabapentin and pregabalin $(52,53)$; human studies showed inhibitory effects of standard analgesic drugs on cortical pain potentials elicited by electrical stimulation (54). Thus, we cannot exclude a small inhibitory influence of long-term use of 


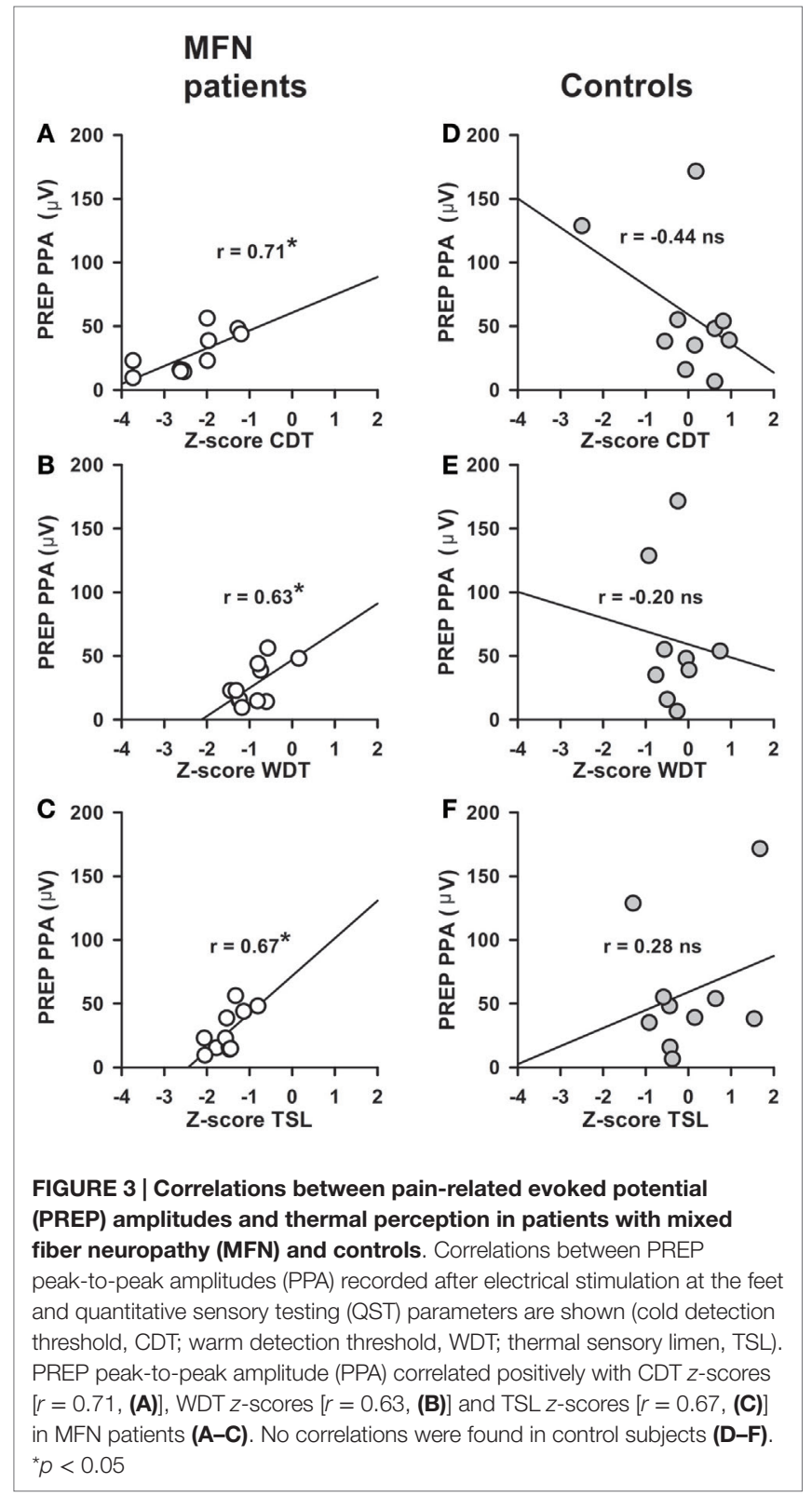

anticonvulsants with analgesic properties on PREP parameter in our study. However, we do not estimate a relevant effect affecting our results as these anticonvulsants are no direct analgesic drugs and do not block nociceptive transmission within the spinal cord. MFN can be characterized by QST in addition to examining nerve conduction and clinical symptoms. Increased mechanical (MDT), thermal (CDT, WDT), and vibration detection thresholds (VDT) imply the impairment of small and large nerve fibers $(28,29)$. The relationship between reduced PREP amplitudes and increased CDT (more pronounced than WDT and TSL) highlights PREP amplitudes as a function of A $\delta$ - more than C-fibers. PREP amplitudes in MFN patients may thus be suitable for clinical application when pharmacotherapy or the MFN time course needs to be evaluated, although these correlations are not turning to account for a single patient. The relationship between PREP amplitudes
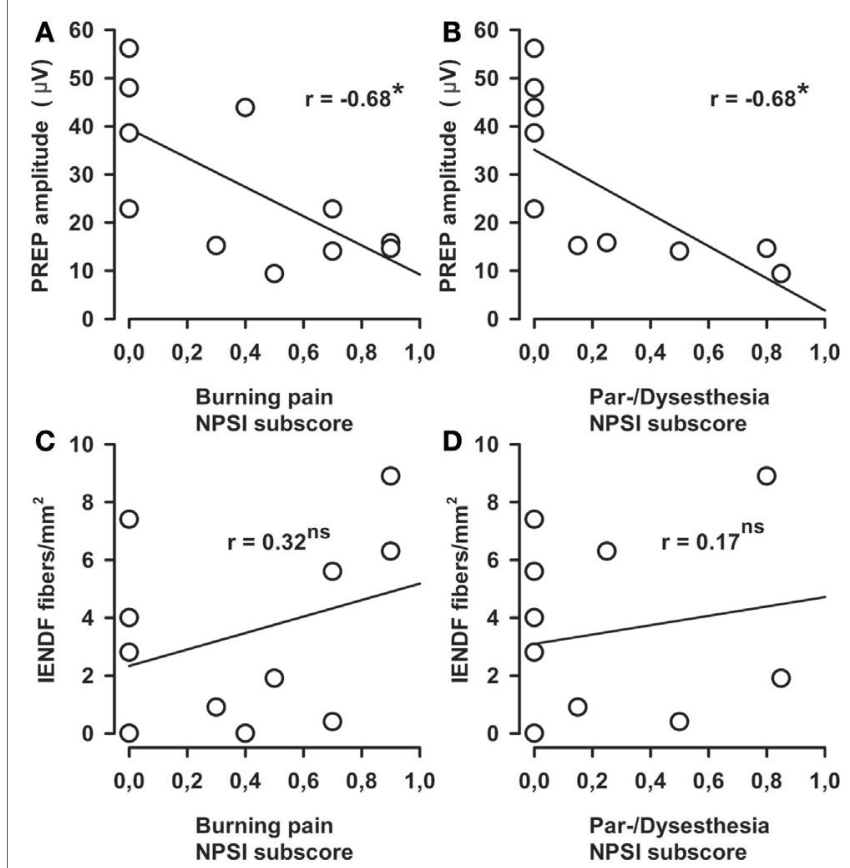

FIGURE 4 | Correlations between burning pain and abnormal sensation as well as pain-related evoked potential (PREP) amplitudes and intraepidermal nerve fiber density. Negative correlation between PREP peak-to-peak amplitudes (PPA) and burning pain in the Neuropathic Pain Symptom Inventory (NPSI) subscore $(r=0.68)$ in mixed fiber neuropathy (MFN) patients is shown in (A). Similar negative correlations appeared between PPA and abnormal sensations in the NPSI subscore $[r=0.68$, (B)]. However, intra-epidermal nerve fiber density (IENFD) and burning pain in (C) and abnormal sensation in the NPSI subscore in (D) revealed no significant correlations. ${ }^{*} p<0.05$

and thermal perception is only detectable under pathological, not healthy conditions. This may indicate that the small fiber degeneration in skin alters both $A \delta$-mediated pain processing and thermal perception mediated by both $\mathrm{A} \delta$ - and $\mathrm{C}$-fibers to a similar degree. Therefore, in pathological conditions like those that MFN patients experience, PREP are sensitive markers of small nerve fiber degeneration. Concordant with these findings, we did not detect any correlations between PREP amplitudes and QST parameters that reflect large fiber dysfunction such as VDT and MDT. Moreover, QST parameters that reveal C-fiber function such as HPT did not correlate with PREP amplitudes. This lack of correlation appears to reveal a potential role of PREP amplitudes in assessing primarily $\mathrm{A} \delta$-fiber function. The correlation between PREP amplitudes and QST parameters reflecting small fiber dysfunction across different MFN subgroups in a small cohort supports PREP as a useful instrument for assessing small fiber dysfunction in addition to QST. Recording PREP at the feet is less time-consuming than the QST standard protocol, which would make easy administration in clinics potentially relevant in followup situations after pharmacotherapy or to evaluate therapeutic efficacy. Furthermore, as disturbances of thermal perception accessible by combining QST and PREP often precede large fiber dysfunction in polyneuropathies $(55,56)$, PREP and QST may make early diagnosis more feasible. 
The absent correlation between skin biopsy results and PREP parameters is in agreement with antecedent reports $(12,13)$. This lack of correlation is most likely based on the fact that PREP predominantly measure A $\delta$ fibers, whereas the intra-epidermal nerve fibers cover mostly C-fibers. In addition, PREP and skin biopsies were not obtained from exactly the same location (IENFD: lower leg, $10 \mathrm{~cm}$ upstream to the lateral malleolus; PREP: foot dorsum).

One reason why burning pain revealed no correlation with the IENFD in MFN patients might be that preserved C-nociceptors may cause spontaneous ectopic discharges resulting in pain (57-59). In a LEP study investigating patients with painful MFN of different origin, an inverse correlation was reported between LEP amplitudes and spontaneous burning pain, while evoked pain did not correlate with LEP (60). Damage to nociceptive fibers that have lost their intra-epidermal terminals was assumed to be the underlying pathophysiological mechanism for the latter (60), which may also apply to our findings.

Different pain phenomena might indicate potential pain mechanisms in MFN patients $(60,61)$. This opens the avenue to improved therapeutic strategies based on mechanisms instead of etiologies. Our cohort tended to complain of burning pain supposedly mediated via C-fibers (62). This finding concurs with the predominance of $\mathrm{C}$-fiber versus $\mathrm{A} \delta$-fiber-mediated pain in our findings from the McGill pain questionnaire in CIDP and vasculitic neuropathy patients (23). It is not surprising that C-fibermediated pain dominates pain sensation in neuropathic pain states, as C-nociceptors demonstrate high spontaneous activity in painful neuropathies, as microneurography demonstrates (59). Furthermore, experimental rat models have shown that abnormal peripheral C-nociceptor discharges with multiple spiking due to altered conduction in nociceptors may account for neuropathic pain (57). In the MFN Guillain-Barré syndrome, IENFD was lower in patients without pain than in those suffering from neuropathic pain (17). This finding supports the notion that preserved C-fibers are major contributors to neuropathic pain in MFN.

\section{REFERENCES}

1. Singleton JR, Smith AG, Bromberg MB. Painful sensory polyneuropathy associated with impaired glucose tolerance. Muscle Nerve (2001) 24:1225-8. doi:10.1002/mus.1136

2. Hoejmakers JG, Faber CG, Lauria G, Merkies IS, Waxman SG. Small-fibre neuropathies-advances in diagnosis, pathophysiology and management. Nat Rev Neurol (2012) 29:369-79. doi:10.1038/nrneurol.2012.97

3. González-Duarte A, Lem-Carrillo M, Cárdenas-Soto K. Description of transthyretin S50A, S52P and G47A mutations in familial amyloidosis polyneuropathy. Amyloid (2013) 20:221-5. doi:10.3109/13506129.2013.827110

4. Zara G, Zambello R, Ermani M. Neurophysiological and clinical responses to rituximab in patients with anti-MAG polyneuropathy. Clin Neurophysiol (2011) 122:2518-22. doi:10.1016/j.clinph.2011.05.015

5. Van den Bergh P, Hadden RD, Bouche P, Cornblath DR, Hahn A, Illa I, et al. European federation of neurological societies/peripheral nerve society guideline on management of chronic inflammatory demyelinating polyradiculoneuropathy: report of a joint task force of the European federation of neurological societies and the peripheral nerve society - first revision. Eur J Neurol (2010) 17:356-63. doi:10.1111/j.1468-1331.2009.02930.x

6. Cruccu G, Sommer C, Anand P, Attal N, Baron R, Garcia-Larrea L. EFNS guidelines on neuropathic pain assessment: revised 2009. Eur J Neurol (2010) 17:1010-8. doi:10.1111/j.1468-1331.2010.02969.x

\section{CONCLUSION}

In summary, our data suggest that PREP amplitudes may be a sensitive and non-invasive marker of small fiber impairment in painful MFN in conjunction with QST. However, it is crucial to endorse that the clinical diagnosis of small fiber impairment in MFN can only be made by performing other small fiber tests in addition to recording PREP. Our findings support PREP amplitudes as an adequate tool to measure small fiber conductivity in patients with MFN or for follow-up after pharmacotherapy. Large-scale future investigations are required to demonstrate the utility of PREP in different subgroups of MFN patients as an instrument for better differential diagnosis and treatment.

\section{AUTHOR CONTRIBUTIONS}

$\mathrm{NH}, \mathrm{NÜ}$, CS wrote the manuscript, designed the study, and undertook statistical analysis; $\mathrm{NH}$ and $\mathrm{AK}$ performed the experiments; CS, DZ, NÜ, NH analyzed data; ZK revised the manuscript for relevant intellectual content.

\section{ACKNOWLEDGMENTS}

We appreciate the cooperation of all study participants. Technical assistance was provided by B. Broll and K. Stahl at the Department of Neurology, University of Würzburg. This publication was funded by the German Research Foundation (DFG) and the University of Würzburg in the funding programme Open Access Publishing.

\section{SUPPLEMENTARY MATERIAL}

The Supplementary Material for this article can be found online at http://journal.frontiersin.org/article/10.3389/fneur.2015.00244

7. Casanova-Molla J, Grau-Junyent JM, Morales M, Valls-Solé J. On the relationship between nociceptive evoked potentials and intraepidermal nerve fiber density in painful sensory polyneuropathies. Pain (2011) 152:410-8. doi:10.1016/j.pain.2010.11.012

8. Treede RD, Lorenz J, Baumgärtner U. Clinical usefulness of laser-evoked potentials. Neurophysiol Clin (2003) 33:303-14. doi:10.1016/j.neucli.2003.10.009

9. Kaube H, Katsarava Z, Käufer T, Diener H, Ellrich J. A new method to increase nociception specificity of the human blink reflex. Clin Neurophysiol (2000) 111:413-6. doi:10.1016/S1388-2457(99)00295-3

10. Mueller D, Obermann M, Koeppen S, Kavuk I, Yoon MS, Sack F. Electrically evoked nociceptive potentials for early detection of diabetic small-fiber neuropathy. Eur J Neurol (2010) 17:834-41. doi:10.1111/j.1468-1331.2009.02938.x

11. Obermann M, Katsarava Z, Esser S, Sommer C, He L, Selter L, et al. Correlation of epidermal nerve fiber density with pain-related evoked potentials in HIV neuropathy. Pain (2008) 138:79-86. doi:10.1016/j.pain.2007.11.009

12. Üçeyler N, Kahn AK, Kramer D, Zeller D, Casanova-Molla J, Wanner $\mathrm{C}$, et al. Impaired small fiber conduction in patients with Fabry disease: a neurophysiological case-control study. BMC Neurol (2013) 13:47. doi:10.1186/1471-2377-13-47

13. Üçeyler N, Zeller D, Kahn AK, Kewenig S, Kittel-Schneider S, Schmid A, et al. Small fibre pathology in patients with fibromyalgia syndrome. Brain (2013) 136:1857-67. doi:10.1093/brain/awt053 
14. Yoon MS, Obermann M, Dockweiler C, Assert R, Canbay A, Haag S. Sensory neuropathy in patients with cryoglobulin negative hepatitis-C infection. $J$ Neurol (2011) 258:80-8. doi:10.1007/s00415-010-5686-1

15. Chiang MC, Lin YH, Pan CL, Tseng TJ, Lin WM, Hsieh ST. Cutaneous innervation in chronic inflammatory demyelinating polyneuropathy. Neurology (2002) 59:1094-8. doi:10.1212/WNL.59.7.1094

16. Martinez V, Fletcher D, Martin F, Orlikowski D, Sharshar T, Chauvin M, et al. Small fibre impairment predicts neuropathic pain in Guillain-Barré syndrome. Pain (2010) 151:53-60. doi:10.1016/j.pain.2010.05.017

17. Ruts L, van Doorn PA, Lombardi R, Haasdijk ED, Penza P. Unmyelinated and myelinated skin nerve damage in Guillain-Barré syndrome: correlation with pain and recovery. Pain (2012) 153:399-409. doi:10.1016/j.pain.2011.10.037

18. Dyck PJ, Sherman WR, Hallcher LM, Service FJ, O'Brien PC, Grina LA, et al. Human diabetic endoneurial sorbitol, fructose, and myo-inositol related to sural nerve morphometry. Ann Neurol (1980) 8:590-6. doi:10.1002/ ana. 410080608

19. Young MJ, Boulton AJM, Macleod AF, Williams DRR, Sonksen PH. A multicentre study of the prevalence of diabetic peripheral neuropathy in the United Kingdom hospital clinic population. Diabetologia (1993) 36:150-4. doi:10.1007/BF00400697

20. Bouhassira D, Attal N, Fermanian J, Alchaar H, Gautron M, Masquelier E. Development and validation of the neuropathic pain symptom inventory. Pain (2004) 108:248-57. doi:10.1016/j.pain.2003.12.024

21. Sommer C, Richter H, Rogausch JP, Frettlöh J, Lungenhausen M, Maier C. A modified score to identify and discriminate neuropathic pain: a study on the German version of the neuropathic pain symptom inventory (NPSI). BMC Neurol (2011) 11:104. doi:10.1186/1471-2377-11-104

22. Stein C, Mendl G. The German counterpart to McGill pain questionnaire. Pain (1998) 32:251-5. doi:10.1016/0304-3959(88)90074-7

23. Beissner F, Brandau A, Henke C, Felden L, Baumgärtner U, Treede RD, et al. Quick discrimination of $\mathrm{A}($ delta) and $\mathrm{C}$ fiber mediated pain based on three verbal descriptors. PLoS One (2010) 5:e12944. doi:10.1371/journal. pone. 0012944

24. Von Korff M, Ormel J, Keefe FJ, Dworkin SF. Grading the severity of chronic pain. Pain (1992) 50:133-49. doi:10.1016/0304-3959(92)90154-4

25. Radloff LS. The CES-D: a self report symptom scale to detect depression in the general population. Appl Psychol Meas (1977) 3:385-401.

26. Hughes R, Bensa S, Willison H, Van den Bergh P, Comi G, Illa I, et al. Randomized controlled trial of intravenous immunoglobulin versus oral prednisolone in chronic inflammatory demyelinating polyradiculoneuropathy. Ann Neurol (2001) 50:195-201. doi:10.1002/ana.1088

27. Collins MP, Dyck PJ, Gronseth GS, Guillevin L, Hadden RD, Heuss D, et al. Peripheral nerve society guideline on the classification, diagnosis, investigation, and immunosuppressive therapy of non-systemic vasculitic neuropathy: executive summary. J Peripher Nerv Syst (2010) 15:176-84. doi:10.1111/j.1529-8027.2010.00281.x

28. Rolke R, Baron R, Maier C, Tölle TR, Treede RD, Beyer A, et al. Quantitative sensory testing in the German research network on neuropathic pain (DFNS): standardized protocol and reference values. Pain (2006) 123:231-43. doi:10.1016/j.pain.2006.01.041

29. Rolke R, Magerl W, Campbell KA, Schalber C, Caspari S, Birklein F, et al. Quantitative sensory testing: a comprehensive protocol for clinical trials. Eur J Pain (2006) 10:77-88. doi:10.1016/j.ejpain.2005.02.003

30. Magerl W, Krumova EK, Baron R, Tölle T, Treede RD, Maier C. Reference data for quantitative sensory testing (QST): refined stratification for age and a novel method for statistical comparison of group data. Pain (2010) 151:598-605. doi:10.1016/j.pain.2010.07.026

31. Mücke M, Cuhls H, Radbruch L, Baron R, Maier C, Tölle T, et al. [Quantitative sensory testing]. Schmerz (2014) 28:635-48. doi:10.1007/s00482-014-1485-4

32. Olson CL. On choosing a test statistic in multivariate analysis of variance. Psychol Bull (1976) 83:579-86. doi:10.1037/0033-2909.83.4.579

33. Magerl W, Wilk SH, Treede RD. Secondary hyperalgesia and perceptual wind-up following intradermal injection of capsaicin in humans. Pain (1998) 74:257-68. doi:10.1016/S0304-3959(97)00177-2

34. Treede RD, Baron R. How to detect a sensory abnormality. Eur J Pain (2008) 12:395-6. doi:10.1016/j.ejpain.2007.11.008

35. Maier C, Baron R, Tölle TR, Binder A, Birbaumer N, Birklein F. Quantitative sensory testing in the German research network on neuropathic pain (DFNS): somatosensory abnormalities in 1236 patients with different neuropathic pain syndromes. Pain (2010) 150:439-50. doi:10.1016/j.pain.2010.05.002

36. Westermann A, Rönnau AK, Krumova E, Regeniter S, Schwenkreis P, Rolke R, et al. Pain-associated mild sensory deficits without hyperalgesia in chronic non-neuropathic pain. Clin J Pain (2011) 27:782-9. doi:10.1097/ AJP.0b013e31821d8fce

37. Kimura J. Electrodiagnosis in Diseases of Nerve and Muscle: Principles and Practice. New York, NY: Oxford University Press (2001).

38. Üçeyler N, Kafke W, Riediger N, He L, Necula G, Toyka KV. Elevated proinflammatory cytokine expression in affected skin in small fiber neuropathy. Neurology (2010) 74:1806-13. doi:10.1212/WNL.0b013e3181e0f7b3

39. Lauria G, Cornblath DR, Johansson O, McArthur JC, Mellgren SI, Nolano M, et al. EFNS guidelines on the use of skin biopsy in the diagnosis of peripheral neuropathy. Eur J Neurol (2005) 12:747-58. doi:10.1111/j.1468-1331.2005.01260.x

40. Pazzaglia C, Vollono C, Ferraro D, Virdis D, Lupi V, Le Pera D. Mechanisms of neuropathic pain in patients with Charcot-Marie-Tooth $1 \mathrm{~A}$ : a laser-evoked potential study. Pain (2010) 149:379-85. doi:10.1016/j.pain.2010.03.001

41. Barraza-Sandoval G, Casanova-Mollá J, Valls-Solé J. Neurophysiological assessment of painful neuropathies. Expert Rev Neurother (2012) 12:1297-309. doi:10.1586/ern.12.93

42. Bragard D, Chen AC, Plaghki L. Direct isolation of ultra-late (C-fibre) evoked brain potentials by $\mathrm{CO} 2$ laser stimulation of tiny cutaneous surface areas in man. Neurosci Lett (1996) 209:81-4. doi:10.1016/0304-3940(96)12604-5

43. Magerl W, Ali Z, Ellrich J, Meyer RA, Treede RD. C- and A delta-fiber components of heat-evoked cerebral potentials in healthy human subjects. Pain (1999) 82:127-37. doi:10.1016/S0304-3959(99)00061-5

44. Iannetti GD, Baumgärtner U, Tracey I, Treede RD, Magerl W. Pinprick-evoked brain potentials: a novel tool to assess central sensitization of nociceptive pathways in humans. J Neurophysiol (2013) 110:1107-16. doi:10.1152/ jn.00774.2012

45. Kakigi R, Endo C, Neshige R, Kuroda Y, Shibasaki H. Estimation of conduction velocity of A delta fibers in humans. Muscle Nerve (1991) 14:1193-6. doi:10.1002/mus. 880141209

46. Lefaucheur JP, Ahdab R, Ayache SS, Lefaucheur-Ménard I, Rouie D, Tebbal D. Pain-related evoked potentials: a comparative study between electrical stimulation using a concentric planar electrode and laser stimulation using a $\mathrm{CO} 2$ laser. Neurophysiol Clin (2012) 42:199-206. doi:10.1016/j.neucli.2011.12.003

47. Katsarava Z, Ayzenberg I, Sack F, Limmroth V, Diener HC, Kaube H. A novel method of eliciting pain-related potentials by transcutaneous electrical stimulation. Headache (2006) 46:1511-7. doi:10.1111/j.1526-4610.2006.00446.x

48. de Tommaso M, Santostasi R, Devitofrancesco V, Franco G, Vecchio E, Delussi $\mathrm{M}$, et al. A comparative study of cortical responses evoked by transcutaneous electrical vs CO(2) laser stimulation. Clin Neurophysiol (2011) 122:2482-7. doi:10.1016/j.clinph.2011.05.006

49. Perchet C, Frot M, Charmarty A, Flores C, Mazza S, Magnin M, et al. Do we activate specifically somatosensory thin fibres with the concentric planar electrode? A scalp and intracranial EEG-study. Pain (2012) 153:1244-52. doi:10.1016/j.pain.2012.03.004

50. Bromm B, Treede RD. Laser-evoked cerebral potentials in the assessment of cutaneous pain sensitivity in normal subjects and patients. Rev Neurol (Paris) (1991) 10:625-43.

51. Cruccu G, Pennisi E, Truini A, Iannetti GD, Romaniello A, Le Pera D, et al. Unmyelinated trigeminal pathways as assessed by laser stimuli in humans. Brain (2003) 126:2246-56. doi:10.1093/brain/awg227

52. Tanabe M, Murakami H, Honda M, Ono H. Gabapentin depresses C-fiberevoked field potentials in rat spinal dorsal horn only after induction of long-term potentiation. Exp Neurol (2006) 202:280-6. doi:10.1016/j. expneurol.2006.06.002

53. Hendrich J, Bauer CS, Dolphin AC. Chronic pregabalin inhibits synaptic transmission between rat dorsal root ganglion and dorsal horn neurons in culture. Channels (2012) 6:124-32. doi:10.4161/chan.19805

54. Rohdewald P, Derendorf H, Drehsen G, Elger CE, Knoll O. Changes in cortical evoked potentials as correlates of the efficacy of weak analgesics. Pain (1982) 12:329-41. doi:10.1016/0304-3959(82)90178-6

55. Jimenez-Cohl P, Grekin C, Leyton C, Vargas C, Villaseca R. Thermal threshold: research study on small fiber dysfunction in distal diabetic polyneuropathy. $J$ Diabetes Sci Technol (2012) 6:177-83. doi:10.1177/193229681200600122 
56. Krøigård T, Schrøder HD, Qvortrup C, Eckhoff L, Pfeiffer P, Gaist D. Characterization and diagnostic evaluation of chronic polyneuropathies induced by oxaliplatin and docetaxel comparing skin biopsy to quantitative sensory testing and nerve conduction studies. Eur J Neurol (2014) 21:623-9. doi:10.1111/ene.12353

57. Serra J, Solà R, Aleu J, Quiles C, Navarro X, Bostock H. Double and triple spikes in C-nociceptors in neuropathic pain states: an additional peripheral mechanism of hyperalgesia. Pain (2011) 152:343-53. doi:10.1016/j.pain.2010.10.039

58. Serra J, Bostock H, Solà R, Aleu J, Garcia E, Cokic B. Microneurographic identification of spontaneous activity in C-nociceptors in neuropathic pain states in humans and rats. Pain (2012) 153:42-55. doi:10.1016/j.pain.2011.08.015

59. Kleggetveit IP, Namer B, Schmidt R, Helås T, Rückel M, Ørstavik K, et al. High spontaneous activity of C-nociceptors in painful polyneuropathy. Pain (2012) 153:2040-7. doi:10.1016/j.pain.2012.05.017

60. Truini A, Biasiotta A, La Cesa S, Di Stefano G, Galeotti F, Petrucci MT, et al. Mechanism of pain in distal symmetric polyneuropathy: a combined clinical and neurophysiological study. Pain (2010) 150:516-21. doi:10.1016/j. pain.2010.06.006
61. Otto M, Bak S, Bach FW, Jensen TS, Sindrup SH. Pain phenomena and possible mechanisms in patients with painful neuropathy. Pain (2003) 10:187-92. doi:10.1016/S0304-3959(02)00313-5

62. Torebjörk HE, Ochoa JL. Specific sensations evoked by activity single identified sensory units in man. Acta Physiol Scand (1980) 110:445-7. doi:10.111 1/j.1748-1716.1980.tb06695.x

Conflict of Interest Statement: The authors declare that the research was conducted in the absence of any commercial or financial relationships that could be construed as a potential conflict of interest.

Copyright (C) 2015 Hansen, Kahn, Zeller, Katsarava, Sommer and Üçeyler. This is an open-access article distributed under the terms of the Creative Commons Attribution License (CC BY). The use, distribution or reproduction in other forums is permitted, provided the original author(s) or licensor are credited and that the original publication in this journal is cited, in accordance with accepted academic practice. No use, distribution or reproduction is permitted which does not comply with these terms. 